\title{
Texture Augmented Detection of Macrophyte Species Using Decision Trees
}

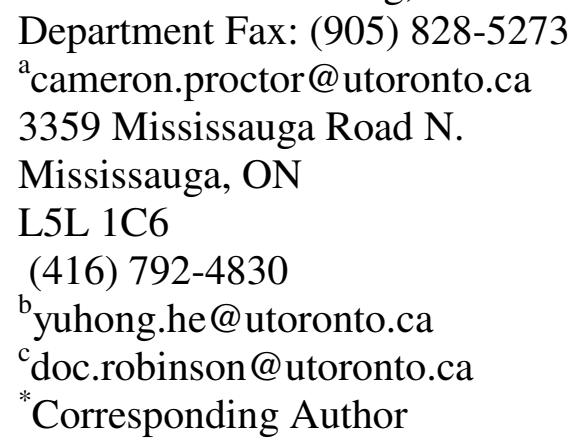

Abstract. Image classification using multispectral sensors has shown good performance in detecting macrophytes at the species level. However, species level classification often does not utilize the texture information provided by high resolution images. This study investigated whether image texture provides useful vector(s) for the discrimination of monospecific stands of three floating macrophyte species in Quickbird imagery of the South Nation River.

Semivariograms indicated that window sizes of $5 \times 5$ and $13 \times 13$ pixels were the most appropriate spatial scales for calculation of the grey level co-occurrence matrix and subsequent texture attributes from the multispectral and panchromatic bands. Of the 214 investigated vectors (13 Haralick texture attributes $* 15$ bands +9 spectral bands +10 transformations/indices), feature selection determined which combination of spectral and textural vectors had the greatest class separability based on the Mann-Whitney U-test and Jefferies-Matusita distance. While multispectral red and near infrared (NIR) performed satisfactorily, the addition of panchromaticdissimilarity slightly improved class separability and the accuracy of a decision tree classifier (Kappa: red/NIR/panchromatic-dissimilarity - 93.2\% versus red/NIR - 90.4\%). Class separability 
31 improved by incorporating a second texture attribute, but resulted in a decrease in classification

32 accuracy. The results suggest that incorporating image texture may be beneficial for separating

33 stands with high spatial heterogeneity. However, the benefits may be limited and must be

34 weighed against the increased complexity of the classifier. 


\section{1. Introduction.}

36 Macrophytes play an important role in many biogeochemical processes such as the

37 production of methane and the fixation of carbon and nitrogen (Matthews \& Fung, 1987; Harden

38 \& Chanton, 1994). However, the services macrophytes perform are likely to change in the near

39 future as climate change is predicted to cause a considerable shift in macrophyte community

40 composition (Root et al., 2003). Specifically, global warming may increase the introduction of

41 invasive species that can alter the physical and chemical characteristics of wetlands and cause

42 significant disturbance that affects populations, communities and ecosystem processes (Benoit \&

43 Askins, 1999). In order to address the many questions regarding macrophyte community

44 composition change and its effects, a reliable and detailed macrophyte monitoring methodology

45 is needed. Field based surveys are often weak in this regard, as they are only practical on

46 relatively small areas (Lee and Lunetta, 1996) and have limited ability to capture the distribution

47 of macrophytes in an entire lake (Zhang, 1998).

As individuals or small macrophyte stands are often of sub-meter to meter extent,

49 monitoring macrophyte via remote sensing can be challenging. Typically the spatial resolution of

50 the sensor must match the size of the target species in order for the species to approach

51 dominance per pixel (Jensen et al. 1993). Previous attempts at species discrimination indicate

52 that the moderate spatial resolution of SPOT and Landsat imagery are insufficient for

53 discrimination at the species level (Armstrong 1993; Zhang 1998; Laba et al., 2008). However,

54 studies focusing on the utility of high spatial resolution (HSR) imagery for multispectral

55 detection of macrophytes demonstrate that good classification accuracy is achievable. Sawaya et

56 al., (2003) revealed that five classes of emergent and four classes of submerged macrophytes

57 could be discriminated from IKONOS imagery with an overall accuracy of $79.5 \%$ and 
58 producer's and user's accuracies from $36 \%$ to $100 \%$. As to be expected, heterogeneous

59 communities with sparse vegetation cover had lower classification accuracy than dense beds of

60 cattail/arrowhead and lily/floating leaf pondweed. At the species level, Dogan et al., (2009)

61 utilized Quickbird imagery to classify three submerged macrophyte classes and two water classes

62 in Lake Mogan, Central Anatolia. Utilizing an unsupervised classification technique, an overall

63 accuracy of $71.69 \%$ was achieved regardless of problems with mixed pixels due to the species

64 being submerged in the water column. In addition, Laba et al., (2008) utilized Quickbird imagery

65 to conduct a land cover classification involving nine species level classes of emergent or floating

66 macrophytes in estuarine ecosystems and coastal watersheds. Classification accuracy ranged

67 from $64.9 \%$ to $73.6 \%$ between four test areas with unique water chemistry (i.e., brackish,

68 oligotrophic and fresh).

69 One of the challenges in utilizing HSR multispectral imagery is that floating macrophyte

70 species have similar biochemical composition such that their spectral signatures may not be

71 sufficiently unique to separate classes (Ge et al., 2006). To respond to the challenges posed by

72 weak spectral variability, many techniques have been developed to utilize the information

73 supplied by image texture. Image texture can be broadly defined as the visual patterns or spatial

74 arrangements of pixels that may have statistical properties, structural properties or both

75 (Haralick, et al., 1973; Borne 1994; Krishnamoorthi \& Seetharaman, 2007). In terms of remotely

76 sensed imagery, texture can be defined as the local brightness variation in a small neighborhood

77 surrounding each pixel (Ross, 2011). Image texture has been associated with many vegetation

78 parameters such as forest-structure variables (age, top height, circumference and basal area)

79 (Kayitakire, et al., 2006; Vincent, et al., 2012), which are often unique to certain vegetation

80 classes, but are not apparent by their spectral signatures. 
The combination of spectral and texture information often outperforms spectral vectors

82 alone (Johansen \& Phinn, 2006). Rao et al., (2002) noted that classification of IRS-1D

83 panchromatic band of the diverse land cover in the Prakasam District of India improved with the

84 incorporation of texture derived using the Grey Level Co-occurrence Matrix (GLCM) approach.

85 Eight popular texture measures derived from a 1-11 pixel window size were semi-qualitatively

86 tested for their capability to discriminate different land cover classes. Utilizing GLCM derived

87 entropy and correlation, an overall classification accuracy of $88.71 \%$ was achieved, which is

88 considerable greater than the $62.89 \%$ achieved by the panchromatic band alone. However, a

89 panchromatic and texture classification further improved the accuracies to $96.24 \%$. Furthermore,

90 textural attributes can be derived from non-optical remote sensing imagery. Arzandeh \& Wang,

91 (2003) derived texture from RADARSAT imagery in a spectral-textural classification

92 monitoring the colonization the Phragmites australis in the cattail dominated Walpole Island in

93 southern Ontario. Incorporation of RADARSAT texture improved the overall accuracy

94 regardless of whether the imagery source was Landsat thematic mapper, Satellite pour

95 l'Observation de la Terre (SPOT) or the Indian Remote Sensing Satellite. Yet the increase in

96 accuracy was minor, with the highest overall accuracy increase only $\sim 3.5 \%$ for the SPOT

97 classification.

98 In terms of texture augmented classification of macrophytes, Laba et al., (2010) reported

99 mixed results in a four species level and seven land cover classification of the Hudson River

100 National Estuarine Research Reserve. A spectral-textural maximum-likelihood classification

101 utilizing variance obtained from a moving window decreased the classification accuracy, often

102 drastically, for the target invasive species classes. Reductions in accuracy were more likely from

103 species that occurred in narrow strips or small patches, suggesting that texture is poorly 
104 representative in these patches due to edge effects and the influence of neighboring patches. In

105 contrast, the results from an object oriented spectral-textural classification were marginally better

106 than a spectral only classification (77.7\% versus $76.2 \%)$. Interestingly the improvement was not

107 systematic, with increases and decreases between the user's and producer's accuracies

108 fluctuating from the spectral classification baseline. For instance, producer's accuracy for $L$.

109 salicaria decreased from 78 to $65 \%$ while user's accuracies increased from 75 to $83 \%$.

110 Moreover the object oriented approach accuracy decreased similarly to the initial moving

111 window approach when a larger $(5 \times 5)$ window is used, suggesting that useful textures are locally

112 defined.

113 However, textural variations between floating macrophyte species have not been

114 examined and it remains in question whether morphological or colonization pattern differences

115 manifest as unique image textures. The aim of this paper is to investigate the utility of a texture

116 augmented classifier to differentiate between stands of three floating macrophyte species. As the

117 texture attribute(s) that improve class separability are unknown, this paper conducts a thorough

118 analysis on a wide range of attributes derived from multispectral, panchromatic, pansharpened,

119 transformed imagery and vegetation indices. Good performing spectral and textural vectors are

120 selected through feature selection that identifies the best combination(s) based on two test of

121 class separability. Combinations of vectors are assessed for overall classification accuracy

122 utilizing a standard training data set and supervised classifier. Since classification results can be

123 affected by differences in scale among the variables, decisions tree classification is utilized as the

124 statistical inferential technique. Decision tree classifiers are an appropriate choice as they are

125 non-parametric rule-based classifiers that can handle nonlinear relations between classes, are

126 easily translatable across scenes and are intuitive (Fayyad \& Irani, 1992). 


\section{2. Methods and Approaches}

\section{$129 \quad 2.1 \quad$ Study area, imagery and field data}

The study area is within a $6 \mathrm{~km}$ section of the South Nation River downstream from the

131 town of Spencerville in the Southeastern Ontario region (Figure 1). As the study area is near the

132 headwaters of the South Nation River, the current is weak and water drains slowly throughout

133 the study area. Bays or inlets off the main water course are partially sheltered, creating pools of

134 water that are partially immobile and stagnant in certain sections. The study area is

135 predominantly comprised of marshland and shallow open water wetlands (National Wetlands

136 Working Group, 1987). Three species of floating macrophytes are present within the study area.

137 The invasive European Frog-bit (Hydrocharis morsus-ranae) is the dominant floating

138 macrophyte, established extensively throughout the study area. Native floating macrophytes

139 Duckweed (Lemma minor) and Yellow Water-lily (Nuphar lutea) are present in limited patches

140 and isolated bays near to shore. As the majority of the littoral zone is colonized by floating

141 macrophytes, submerged macrophytes Coontail (Ceratophyllum demersum) and Floating-leaf

142 Pondweed (Potamogeton natans) are often concealed from the canopy, occurring to a limited

143 extent in stagnant water. Terrestrial species along the riparian zone are a mixture of Common

144 Reed (Phragmities australis), Narrowleaf Cattail (Typha angustifolia) and other grasses and

145 mixed forest predominantly comprised of deciduous species common to Southeastern Ontario

146 such as Red Maple (Acer rubrum), Eastern White Cedar (Thuja occidentalis), Paper Birch

147 (Betula papyrifera), etc. The classification schema (Table 1) is based upon the species noted

148 during the field survey. Three classes are species level classes (contain a single vegetation

149 species) and the remaining land cover types are comprised of multiple species or no vegetation. 
152 product was purchased given the minimal elevation relief of the study area and limited potential 153 for geometric distortions due to elevation change. Atmospheric correction was performed 154 utilizing PCI Geomatica 10 ATCOR2 (PCI Geomatics, 2010) to remove the influence of 155 atmospheric effects on the spectral signal. The correction assumed a high water vapour content 156 since wetlands typically feature abundant atmospheric water content $\left(+3 \mathrm{~g} / \mathrm{cm}^{2}\right)$ and high 157 variability $\left(1-6 \mathrm{~g} / \mathrm{cm}^{2}\right)$ (Guzzi \& Rizzi, 1984). A field survey was conducted between September 4th and 7th, 2010 to collect

159 prototypical ground truth for use as training data. Training data consisted of $1 \mathrm{x} 1 \mathrm{~m}$ quadrats 160 samples obtained by following the point intercept method for macrophyte monitoring guidelines 161 (Alberta Environment, 2006). Sample sites were pre-selected at random in a 50x50m grid pattern 162 prior to the field survey. Quadrats lacking a predominant class with over $80 \%$ of the total canopy 163 cover were excluded. Class labels were assigned to each sample based on the predominant class 164 within the quadrat. The labels assigned matched the colonization patterns documented by local 165 experts (South Nation Conservation Authority) who indicated that the study area was stable and 166 the invasive species European Frog-bit had been dominant each year for the past ten years. Since 167 the species present were likely in equilibrium with their environment, the current distribution of 168 species was likely due to environmental determinants (water depth, current speed) that were not 169 expected to shift significantly over the temporal lag. Furthermore, the data set was previously 170 used by Proctor et al., (2012) in a land cover classification achieving an overall classification 171 accuracy of $84.3 \%$. The samples collected were split approximately $70: 30$ into a training and 172 reference data set (Table 2). Complete details on the collection and processing of training data 
173 can be found in (Proctor, et al., 2010).

$175 \quad 2.2 \quad$ Spectral variables under investigation

To comprehensively investigate whether image texture could improve classification

177 accuracy, textural attributes derived from pansharpened, select vegetation indices and spectral 178 transformation are investigated. Pansharpening of the multispectral bands is assessed as image

179 textures are plausibly more apparent in higher spatial resolution imagery, especially when texture

180 depends upon small canopy gaps. In addition, a number of commonly derived vegetation indices

181 are arbitrarily examined under the notion that the indices would better express the image texture

182 through enhancing the greenness of the vegetation or reduce the influence of the background on

183 the spectral signal. This reasoning is similar for the two spectral transformations. Tasseled cap

184 transformation is examined in order to enhance brightness, greenness or wetness, while principal

185 components examine textures related to variance. These transformations are commonly utilized

186 to improve classification accuracy and could theoretically be utilized to improve the derivation

187 of texture.

189 conduct the tasseled cap transformation. The tasseled cap coefficients were modified from the 190 defaults to those suggested by the comparison paper by Yarbrough, et al., (2005). Pansharpening 191 was performed on all four multispectral bands using the PCI Pansharp tool. PCI was also utilized

192 to conduct the principal components analysis. The vegetation indices, Normalized Difference

193 Vegetation Index (NDVI) (Rouse et al., 1973), Modified Soil Adjusted Vegetation Index

194 (MSAVI) (Qi et al., 1994), Difference between Vegetation and Water (DVW) (Gond et al., 195 2004) and Normalized Difference Water Index of Mc Feeters (NDWIF) (Mc Feeters, 1996) were 
196 calculated in PCI modeller. In total 214 vectors were scrutinized (Table 3).

$198 \quad 2.3 \quad$ Identification of appropriate window size using semivariograms

An important factor that has substantial impact on the derivation of texture is the spatial

200 autocorrelation of pixel values (Franklin, Wulder, \& Lavigne, 1996). Since the degree of spatial

201 autocorrelation is not readily apparent, a simple means to quantify the spatial relationship is to

202 calculate the semivariance. The semivariogram range and sill indicate the distance at which

203 pixels are no longer correlated, beyond which there is no spatial dependence among the

204 reflectance values. Research has shown that the window size used for texture analysis should not

205 exceed the distance represented by the semivariogram range (Franklin et al., 1996). However,

206 each class may be spatially independent and exhibit a unique range of spatial autocorrelation

207 (Treitz \& Howarth, 2000). As a sole window size is utilized to derive texture attributes, the

208 decision on the appropriate window size should consider class variations.

209 Seven image subsets were derived from the imagery in order to determine the

210 semivariance for each class. Each image subset contained a dominant stand of the species within

211 an area of minimal ecosystem complexity. Semivariograms were calculated for the Quickbird

212 multispectral and panchromatic bands where the lag separation distance coincided with the data

213 spacing and the number of lags was set to one-third the diagonal distance of the data matrix.

214 Using the gstat package of R (R Development Core Team 2008), all semivariograms were fitted

215 with a variogram model to provide a more robust representation of the decay in spatial

216 autocorrelation that is less sensitive to noise. A spherical variogram model was chosen because

217 of its commonality in the literature and it resulted in a generally lower sum of squared error

218 when tested against the Gaussian, exponential and circular models. All semivarograms were 
219 fitted with a standard spherical model with the optimal model parameters attuned to minimize the

220 sum of squared error between the fitted model and the measured semivariance. The resulting

221 coefficients of the variogram model were used to inform the window size decision.

\section{$2232.4 \quad$ Deriving texture attributes using the grey level co-occurrence matrix}

224 In the literature, a common approach to determine the image texture is based on the Grey

225 Level Co-occurrence Matrix (GLCM) method developed by (Haralick et al., 1973). Calculating

226 the Haralick texture attributes consists of two parts. The first step is the construction of the co-

227 occurrence matrix. A co-occurrence matrix is a probability matrix whose elements are indexed

228 by gray-level values for all pixel-pairs at a defined distance and angle in a pixel neighborhood.

229 For isotropic texture attributes, co-occurrence matrixes are generated for the four directions of

230 adjacency $\left(0^{\circ}, 45^{\circ}, 90^{\circ}\right.$ and $\left.135^{\circ}\right)$ and the average occurrence is retained for texture derivation

231 (Anys \& He, 1995). The second step utilizes the statistical properties of the co-occurrence

232 matrices to derive the actual texture attributes. Homogeneity, contrast, dissimilarity and entropy

233 are commonly cited as having the best performance (Johansen \& Phinn, 2006). As the image

234 texture of floating macrophytes has not been examined, this study opted to examine 13 texture

235 attributes (Table 4).

236 Since computing the co-occurrence matrix is a computationally intensive task, the values

237 of each vector were rescaled by a linear function. Hence, the co-occurrence matrix was

238 calculated as a square matrix of dimension $\mathrm{Ng}$, where $\mathrm{Ng}$ was the total number of gray levels (32

239 levels) in the image. The matrix was populated by counting the total occasions a pixel with value

$240 i$ was adjacent to a pixel with value $j$, storing the count in the $(i, j)$ th element of the matrix and

241 then subsequently dividing the matrix by the total number of such comparisons that were made. 
242 Hence, the $(i, j)$ th entry in a normalized GLCM probability density matrix was considered as a

243 probability $p(i, j)$ that a pixel with value $i$ was adjacent to a pixel of value $j$, in which adjacency

244 was defined as directionally invariant. For texture attributes based on the Grey Level Difference

245 Vector (GLDM), the matrix stores the count of the absolute difference between the reference

246 pixel and its neighbour expressed as the probability density $p \delta(i, j)$. Each of the 13 texture

247 attributes were calculated for the Quickbird multispectral and panchromatic bands, pansharpened

248 bands and multispectral derived image transformations and vegetation indices (Table 3).

\section{$250 \quad 2.5 \quad$ Feature selection of spectral and texture vectors}

251 Feature selection is a technique for selecting a subset of vectors that consistently provide

252 good performance in class discrimination. All vectors are rank ordered in terms of performance

253 and sequentially trimmed until the relevant vectors providing the greatest class separability

254 remain. The performance of each combination of vectors is assessed via a distance measure.

255 However, a number of distance measures are available and no single index can reliably indicate

256 which vectors are optimal. We therefore employ a feature selection process based on two tests of

257 separability performance utilizing a standard training data set of prototypical ground truth.

258 In the first test, the Mann-Whitney U-test (Sawilowsky, 2007) is a nonparametric

259 approach that does not assume a normal distribution when assessing whether the differences in

260 the medians between class pairs are statistically significant. For each vector, the distributions of

261 two classes are examined. If they are sufficiently intermingled, such that their medians are

262 similar and there is a high degree of overlap, then the class pair is deemed to come from the same

263 population. Hence, the Mann-Whitney U-test indicates the number of class pairs out of the 21

264 possible that are separable on the basis of a single vector 
266 classes are smaller than between them. To tests this hypothesis $\left(H_{1}\right)$ that there is no significant

267 difference between the medians $\eta$ of a class pair, Mann-Whitney U-test examined for $c, c+1$

268 classes and vector $i$ if:

$$
H_{1}: \eta_{c}(i)=\eta_{c+1}(i)
$$

269 At significance level of a $\propto=0.01$ the null hypothesis was rejected and the alternative hypothesis

270 that the medians are not equal was accepted. For each vector, the number of statistically

271 significant class pairs with different medians was tabulated, with vectors approaching 21 class

272 pairs considered useful for class separability.

273 The Mann-Whitney U-test also provides a numerical measure (the U-Statistic) that scales

274 positively with the degree of separability between the medians of a class pair. The U-Statistic is

275 given as the minimum $U$ value calculated independently for each class. The $U$ value for a class is

276 based on the number of pairs for which the value of the first class is less than the second class

277 after being placed in ascending order. When two classes are completely separate, one class

278 always out ranks the other, hence its rank sum is the maximum possible while the other has a

279 zero $U$ value. Consequently the U-Statistic in the minimum, zero indicating high class

280 separability. Conversely, when both groups are interpenetrated the rank sums are similar as the 281 class that out ranks the other fluctuates. Hence, the U-Statistic is the maximum which is half of 282 the number of training data samples, $n_{c}$ times $n_{c+1}$.

283 For each class pair, the Mann-Whitney U-Statistic was calculated as:

$$
\begin{gathered}
U=\min \left(U_{c}, U_{c+1}\right) \\
U_{c}=n_{c} n_{c+1}+\frac{n_{c}\left(n_{c}+1\right)}{2}-R_{c}
\end{gathered}
$$




$$
U_{c+1}=n_{c} n_{c+1}+\frac{n_{c+1}\left(n_{c+1}+1\right)}{2}-R_{c+1}
$$

284 where $U_{c}, U_{c+1}$ are the $U$ values calculated for classes $c, c+1, n_{c}, n_{c+1}$ are the number of 285 training data samples for each class and $R_{c}, R_{c+1}$ are the sum of the ranks for classes $c, c+1$.

286 As the U-Statistic was scaled from 0 to $n_{c} * n_{c+1}$ it was subsequently normalized between 0 and 2871 by:

$$
U_{n o r m}=\frac{n_{c} n_{c+1}}{2}-\frac{U}{\frac{n_{c} n_{c+1}}{2}}
$$

The second test evaluates class separability based on the Jefferies-Matusita distance 289 (Richards, 1993) for a combination of vectors. The Jefferies-Matusita distance is widely used to 290 identify the band combination that yields the best spectral separability between two classes 291 (Adam \& Mutanga, 2009). There are multiple strategies to extend the Jefferies-Matusita distance 292 to the multi-class case (Bruzzone et al., 1995) with the simplest accomplished by calculating the 293 distance between all 21 class pairs and calculating the average distance.

294 The similarity of two class density functions was measured by the Bhattacharya distance 295 defined as follows:

$$
b=\frac{1}{8}\left(\mu_{2}-\mu_{1}\right)^{T}\left[\frac{\sum_{1}-\sum_{2}}{2}\right]^{-1}\left(\mu_{2}-\mu_{1}\right)+\frac{1}{2} \ln \frac{\left|\left(\sum_{1}-\sum_{2}\right) / 2\right|}{\left|\sum_{1}\right|^{\frac{1}{2}}\left|\sum_{2}\right|^{\frac{1}{2}}}
$$

296 where $\mu_{i}$ and $\sum_{1}$ are the mean vector and covariance matrix of class $i$, respectively.

297 Since Bhattacharya distance increases exponentially it was converted to the Jefferies-

298 Matusita distance which has a saturating behavior with increasing class separation and is

299 asymptotic to the value 2. The Jefferies-Matusita distance was calculated as:

$$
J M=2 *\left(1-e^{-b}\right)
$$


A Jefferies-Matusita distance greater than 1.0 indicates the two classes are very similar

301 and thus hard to separate. Values between 1.00 and 1.9 indicate that the two classes could be

302 separated at least to some extent while values greater than 1.9 are considered to yield good to

303 excellent separation.

$304 \quad$ Calculating the Jefferies-Matusita distance for all possible band combinations was not

305 feasible given the number of potential permutations. In order to save computation time,

306 sequential forward selection was used as opposed to an exhaustive search of each vector

307 combination (Gruninger et al., 2001). Forward selection utilizes an interactive process to identify

308 the best performing vector and sequentially adds additional vectors until the improvement in

309 performance declines. Hence, the first iteration selected the best single vector based upon

310 Jefferies-Matusita distance. This vector was then paired with every remaining vector to find the

311 best two vector combination based upon the Jefferies-Matusita distance. Repeating the process,

312 the vector combination was subsequently augmented until additional vectors contributed less

313 than 0.01 to class separability.

\section{$3152.6 \quad$ Decision Trees using selected features.}

316 A classification and regression tree classifier implemented in the rpart package of $R$ ( $R$

317 Development Core Team 2008) was utilized to classify each vector combination identified

318 through feature selection. All relevant vectors were appended to a training data set of $\sim 500$

319 samples of labelled class based on the sample location. In the supervised decision tree approach,

320 vectors were predictor variables mapped to the target classes subject to a minimized cost

321 function (Xu et al., 2005). The algorithm was conditioned to select the split that partitions the

322 data into parts such that the sum of the squared deviations from the mean in the separate parts 
323 was minimized. To minimize over fitting, a pruning process was adopted to remove surplus

324 terminal nodes that provide little power to classify instances and may have been based on noisy

325 or erroneous data. Selection of the surplus terminal nodes was guided by the cost-complexity

326 parameter, which is a measure of average error reduced per terminal nodes. The pruned tree was

327 used to predict the class of all unknown pixels in the imagery.

$329 \quad 2.7 \quad$ Classification accuracy

330 For the final classification, the accuracy was assessed utilizing a standard error matrix

331 (Congalton, 1991; Congalton, \& Green, 1999). The global classification accuracy metrics were

332 the overall classification accuracy and kappa coefficient. The overall accuracy was the number of

333 correctly classified pixels over the number of pixels utilized in the accuracy assessment. As a

334 number of pixels were likely allocated to the correct class by chance, the kappa coefficient was

335 used to determine the classification accuracy after chance has been accounted for. The measures

336 of class accuracy were the user's accuracy (UA) and producer's accuracy (PA) derived from the

337 error matrix. UA was calculated as the percentage of pixels correctly assigned to a class out of

338 the total pixels classified as said class. PA was calculated as the percentage of pixels correctly

339 assigned to a class out of the total pixels of said class utilized in the accuracy assessment.

341 3. Results

\section{$342 \quad 3.1 \quad$ Semivariogram Analysis}

343 Semivariance for the multispectral bands generally began to saturate at a lag of six pixels.

344 However, per class semivariograms indicate that certain classes had unique spatial

345 autocorrelation. The Mixed Forest (MF) class has a considerable shorter range of spatial 
346 autocorrelation (3.25 pixels, 7.8m) than the Grasses, Sedges, Rushes (GSR) class (8.5 pixels,

$34720.4 \mathrm{~m})$. The lower range of spatial autocorrelation for the MF class is likely the result of canopy

348 gaps between individual trees. Comparing the floating macrophytes, Fragrant Water-lily (FWL)

349 spatial autocorrelation (Figure 2a) is shorter than European Frog-bit (EFB) (Figure 2b) which

350 forms denser tessellated canopies. Examination of FWL patches revealed they rarely exceeded

35110 pixels in size and were always surrounded by open water. In contrast, EFB patches are linear,

352 stretching 100 of meters along the shoreline and extending $>10 \mathrm{~m}$ from shore, if not completely

353 covering the water surface. Considering the considerable contrast between FWL and the

354 surrounding open water, FWL stands may have a greater range in grey levels provided the

355 window is sufficiently large to capture the surrounding water column. Hence the panchromatic

356 band may be more appropriate to derive image texture than the multispectral bands. The level of

357 spatial detail captured is greater and the difference in spatial autocorrelation range between EFB

358 and FWL is greater. Considering the mean and mode in the semivariance range of all classes

359 (Table 5), these results indicate that a window size of 5x5 is more appropriate for the

360 multispectral bands and 13x13 for the panchromatic band.

361

\section{$362 \quad 3.2 \quad$ Selected Features}

363 Out of 214 vectors examined, 154 have statistically significant Mann-Whitney U-test

364 results which considered useful for class separability (defined as >19 out of 21 statistically

365 significant class pairs) (Figure 3). The multispectral Quickbird bands perform well, eliciting both

366 a high number of class pairs and U-Statistic. The pansharpened multispectral bands have a

367 slightly reduced performance, possibly due to color distortion from the pansharpening process.

368 The top performing vectors are generally the transformed vectors and vegetation indices. Indices 
369 such as NDVI slightly outperformed the sole spectral bands, as to be expected from the higher

370 information content and close relationship of NDVI to vegetation biochemistry. In particular, the

371 Normalized Difference Water Index had the highest U-Statistic (0.908), slightly higher than all

372 spectral bands (blue: 0.815, green: 0.883, red: 0.895, NIR: 0.831).

373 Texture attributes have generally lower performance than the baseline provided by the

374 spectral bands. Fewer class pairs are statistically significant and the U-Statistic is noticeably

375 reduced (Figure 3). The texture attribute Mean is the exception, with comparable performance if

376 derived from the multispectral NIR band or Tasseled Cap brightness ( 0.871 and 0.892

377 respectfully). It is important to note that Mean texture is a descriptive statistic based on the

378 frequency of occurrence of a value in combination with a certain neighbour pixel value. Mean

379 texture thus differs from the mean pixel value within a window, but shares some similarities.

380 Other high performing texture attributes are contrast, dissimilarity and entropy. There is little

381 difference between entropy and its related cousin, GLDV entropy.

382 The performance of a texture attribute is strongly related to its derivative source. Texture

383 derived from multispectral band 1 and the $2^{\text {nd }}$ or $3^{\text {rd }}$ principal components consistently performed

384 poorly, regardless of the texture attribute derived. In contrast, texture derived from the

385 panchromatic band often outpreformed all other sources. For instance, dissimilarity derived from

386 the panchromatic band has 20 out of 21 statistically significant class pairs and a high U-Statistic

387 (0.84). The next highest performing dissimilarity texture vector is derived from the Tasseled Cap

388 brightness band (21 significant class pairs, U-Statistic 0.77 ). The performance of panchromatic

389 derived texture for certain texture attributes is comparable to the poorer performing spectral

390 bands. These results indicate some potential utility for image texture in combination with the

391 spectral bands. 
The second test based on Jefferies-Matusita distance employing a forward selection

393 search indicates increasing class separability as additional vectors are utilized up until four

394 vectors (Figure 4). Beyond four vectors the increase in Jefferies-Matusita distance is negligible.

395 Based on the average Jefferies-Matusita distance between all class pairs, the first iteration of the

396 forward selection search identifies multispectral red as vector providing greatest class

397 separability. As expected for a sole band, class separability between class pairs ranges

398 considerably and the overall Jefferies-Matusita distance is a poor 1.43. The second iteration

399 recalculated the Jefferies-Matusita distance for all combinations of the multispectral red vector

400 and an additional vector. The second vector, multispectral NIR, improves class separability

401 considerably (Table 6). The average Jefferies-Matusita distance of 1.87 approaches the good-

402 excellent separation threshold. Hence, the spectral bands alone are likely sufficient to achieve

403 satisfactory classification accuracy. Additional iterations identified the texture attributes,

404 panchromatic dissimilarity and Tasseled Cap band 1 contrast as vectors improving class

405 separability. Incorporation of panchromatic dissimilarity increased the Jefferies-Matusita

406 distance to 1.97. An additional fourth vector (Tasseled Cap band 1 contrast) negligibly improves 407 separability.

408 Both test results indicate that augmenting the spectral vectors with an image texture yield

409 a slight improvement in class separability that may translate into improved classifier

410 performance. To examine this premise, the band combination results from iteration 2-3 are

411 classified. Thus, a spectral only classification involving two bands is compared to two

412 classifications augmenting the spectral vectors with image texture, one involving two texture

413 bands. 


\section{$415 \quad 3.3 \quad$ Decision Tress Classification}

416 Visually the three classifications are similar and colonization patterns of each floating

417 macrophyte species generally match the patterns noted in the field (Figure 5). The Submerged

418 (Sub) class has a slightly greater extent in the third iteration while patches of Duckweed (DW)

419 are smaller and more fragmented. The salt and pepper pattern of misclassified pixels within the

420 forested areas is also reduced. The fourth iteration has noticeably increased the prevalence of

421 misclassified terrestrial pixels. There is also greater confusion between the MF and FWL classes.

422 The pruned decisions trees for the suite of vectors from the second and third iterations

423 had similar nodes for the majority of the tree (Figure 6). The land cover classes MF, GSR and

424 Open Water (OW) were similarly classified on the basis of the NIR and red bands. The decision

425 trees diverge in the classification of SUB, FWL and GSR. The Red/NIR only decision tree

426 utilizes the NIR band to separate the SUB land cover class. In contrast, the texture augmented

427 decision tree utilizes panchromatic dissimilarity for the same task. In both cases FWL and GSR

428 are separated by the red band. The four vector decision tree is markedly different with only the

429 first node in common. Texture is utilized further up the tree at the second and third node level.

\section{$431 \quad 3.4 \quad$ Classification Accuracy}

432 The overall classification accuracy of the three decision tree classifiers is high. Each

433 classifier performed comparably well in terms of accuracy for the land cover classes. Accuracies

434 for these classes are $>90 \%$ and stable between classifiers. However, performance in classifying

435 the three species level varied slightly. Incorporation of image texture slightly improved the

436 accuracy for FWL for iteration 3, but not iteration 4 (Table 7).

437 While iteration 4 saw a slight improvement in accuracy for other classes, the accuracy for 
438 the FWL class is reduced compared to the second and third iteration. The incorporation of a

439 second texture band appears to have a negative effect on classifying the species level classes and

440 the overall accuracy. While Kappa increased with the third iteration (second iteration 90.4 versus

441 third iteration 93.0) the fourth iteration saw a decrease (fourth iteration 89.8). Hence the

442 classification accuracy peaked for the decision tree classification involving panchromatic

443 dissimilarity. Incorporation of additional textures may increase the classification complexity,

444 resulting in decreased accuracy.

\section{4. Discussion and Conclusions}

447 The results suggest that the practical value of texture augmented classification for floating 448 macrophyte species discrimination is low. The slight increase in accuracy comes at a large cost 449 in terms of computational complexity and time requirements. Furthermore, suitable image 450 textures are sensitive to their parameterization. Considerable variations in the efficacy of the 451 texture attributes contrast and dissimilarity were noted between a GLCM of 32 and 256 levels, 452 with the lower number of grey levels yielding better performance. Hence, there is considerable 453 trial and error in the process of identifying a suitable texture attribute and the results may be case 454 specific. Considering the slight benefits versus the increased resource cost, the payback of a 455 texture augmented classifier is unclear.

456 However, the hypothesis that canopy gaps in certain species of floating macrophytes would 457 manifest as detectable differences in texture may have some merit. The study results indicate a 458 greater tendency in texture attributes such as contrast and dissimilarity, which respond to 459 heterogeneity in land cover and complex spatial structures, to have some utility in improving 460 class separation and in land cover classification. In the case of FWL, the surrounding open water 
461 produced a noticeable texture that could be utilized to reduce its confusion with submerged

462 macrophytes. This result suggests that image texture in floating macrophyte stands has a stronger

463 association with spatial heterogeneity and canopy gaps than an internal systemic texture. Hence,

464 the number of open water cells is the surrounding area maybe a simpler means to express texture

465 in this instance.

466 These findings can also be interpreted in the context of other studies on image texture of

467 wetland vegetation. Laba et al., (2010) reported that texture augmented classification utilizing 4

468 texture bands in addition to the IKONOS multispectral bands reduced accuracy approximately

$46930 \%$ versus the baseline multispectral only classification. Ultimately the overall classification

470 accuracy was slightly improved by incorporating texture (77.7\% with texture versus $76.2 \%$

471 without) using an edge-preserving spectral-smoothing-segmentation procedure. However, the

472 improvements were not across the board. In many cases accuracies decreased for certain classes

473 in lockstep with accuracy increase in other classes. Overall no class had major improvements

474 relative to the accuracies found for baseline method. Our findings are in line with those of Laba

475 et al., (2010).

476 As demonstrated by Laba et al., (2010) the contribution of image texture may depends upon

477 the methodology. It is plausible that more robust methodologies to derive image texture may

478 yield greater improvements in performance, especially methodologies that account for the

479 tendency of macrophytes to occur in complex and narrow patches such those that occur along

480 shorelines. In addition, the spectral similarity between the classes of interest may influence the

481 relative contribution of image texture. As the classification accuracy of this study utilizing

482 spectral only variables was high, the need for additional vectors was low. Image texture is more

483 likely to yield a greater contribution when discriminating between macrophytes with highly 
484 similar spectral signatures, but contrasting biophysical attributes.

Factors such as spectral and spatial resolution may be more related to the classification

486 accuracy than image texture. Using a ground and airborne hyperspectral sensor, Malthus \&

487 George, (1997) noted clear spectral differences between 11 species in the Cefni Reservoir, UK.

488 Utilizing only 3 bands a 79\% classification accuracy was achieved. In terms of spatial resolution,

489 often higher resolution yields more inner class complexity, decreasing the final accuracy.

490 Pasqualini et al., (2005) documented lower accuracy (73\%) when utilizing pansharpened $2.5 \mathrm{~m}$

491 Spot 5 imagery for seagrass detection versus the non-pansharpened 10m imagery (96\%).

492 Classification methodology can also subtly influence the final accuracy. Slightly lower

493 classification accuracies for this study site in a similar land cover classification were reported by

494 Proctor, et al., (2010). The fuzzy support vector machines classification methodology yielded

$49587.4 \%$ while a fuzzy c-means classifier yielded a $72.8 \%$ classification accuracy. The difference

496 between the three classification methodologies is much larger than the additional accuracy

497 provided by image texture, suggesting that a suitable processing technique for extracting spectral

498 information of wetland vegetation maybe more linked to the overall classification accuracy than

499 the incorporation of image texture.

501 Acknowledgments.

502 Many funding agencies have provided funding for this research. The partial support of Discovery

503 Grant RGPIN-44611 and RGPIN-386183 from the Natural Sciences and Engineering Research

504 Council (NSERC) is gratefully acknowledged. We also thank the Department of Geography of

505 the University of Toronto Mississauga for funding from the Graduate Expansion Fund to cover

506 the costs of field work and conference attendance. 


\section{References}

Adam, E., Mutanga, O. 2009. Spectral discrimination of papyrus vegetation (Cyperus papyrus L.) in swamp wetlands using field spectrometry. ISPRS Journal of Photogrammetry and Remote Sensing, 64 (6), 612-620.

Alberta Environment. 2006. Aquatic ecosystems field sampling protocols Retrieved 7/10/2011, 2011, from http://environment.gov.ab.ca/info/posting.asp?assetid=7805\&categoryid=5

Anys, H., He, D. 1995. Evaluation of textural and multipolarization radar features for crop classification. IEEE Transactions on Geoscience and Remote Sensing, 33(5), 1170-1181.

Arzandeh, S., Wang, J. 2003. Monitoring the change of phragmites distribution using satellite data. Canadian Journal of Remote Sensing, 29(1), 24-35.

Bajjouk, T., Guillaumont, B., \& Populus, J. 1996. Application of airborne imaging spectrometry system data to intertidal seaweed classification and mapping. Hydrobiologia, 326-327, 463-471.

Benoit, L. K., Askins, R. A. 1999. Impact of the spread of phragmites on the distribution of birds in connecticut tidal marshes. Wetlands, 19(1), 194-208.

Borne F. 1994. New Features for Texture Analysis. In: editors (Eds.), Surface and Atmospheric Remote Sensing: Technologies, Data Analysis and Interpretation, International Geoscience and Remote Sensing Symposium IGARSS'94 Proceedings, CalTech, Pasadena, California, August 812.

Bruzzone, L., Roli, F., Serpio, S.B. 1995 An extension of the Jeffreys-Matusita Distance to Multiclass Cases for Features Selection. IEEE Transactions on Geoscience and Remote Sensing, 33(6), 1318-1321.

Congalton, R. 1991. A review of assessing the accuracy of classifications of remotely sensed data. Remote Sensing of Environment, 37(1), 35-46.

Congalton, R., Green, K. 1999. Assessing the accuracy of remotely sensed data: Principles and practices. Lewis Publications.

DigitalGlobe. 2005. Quickbird scene 052421306010_01_P001, level standard 2A. Longmont, Colorado: DigitalGlobe.

Fayyad, U. M., Irani, K. B. 1992. On the handling of continuous-valued attributes in decision tree generation. Machine Learning, 8(1), 87-102.

Franklin, S. E., Wulder, M. A., \& Lavigne, M. B. 1996. Automated derivation of geographic window sizes for use in remote sensing digital image texture analysis. Computers and 
Geosciences, 22(6), 665-673.

Ge, S., Everitt, J., Carruthers, R., Gong, P., \& Anderson, G. 2006. Hyperspectral characteristics of canopy components and structure for phenological assessment of an invasive weed. Environmental Monitoring and Assessment, 120(1-3), 109-26.

Gond, V., Bartholome, E., Ouattara, F., Nonguierma, A. \& Bado, L. 2004. Surveillance et cartographie des plans d'eau et des zones humides et inondables en régions arides ave l'instrument VEGETATION embarqué sur SPOT-4. International Journal of Remote Sensing, 25(5), 987-1004.

Gruninger, J., Sundberg, R., Fox, M., Levine, R., Mundkowsky, W., Salisbury, M. S., \& Ratcliff, A. H. 2001. Automated optimal channel selection for spectral imaging sensors. 4381 68-75.

Guzzi, R., \& Rizzi, R. 1984. Water vapor absorption in the visible and near infrared: Results of field measurements. Applied Optics, 23(11), 1853-1861.

Haralick, R. M., Shanmugam, K., \& Dinstein, I. 1973. Textural features for image classification. IEEE Transactions on Systems, Man and Cybernetics, smc 3(6), 610-621.

Harden, H. S., Chanton, J. P. 1994. Locus of methane release and mass-dependent fractionation from two wetland macrophytes. Limnology and Oceanography, 39(1), 148-154.

Johansen, K., Phinn, S. 2006. Linking riparian vegetation spatial structure in australian tropical savannas to ecosystem health indicators: Semi-variogram analysis of high spatial resolution satellite imagery. Canadian Journal of Remote Sensing, 32(3), 228-243.

Kayitakire, F., Hamel, C., \& Defourny, P. 2006. Retrieving forest structure variables based on image texture analysis and IKONOS-2 imagery. Remote Sensing of Environment, 102(3-4), 390401.

Krishnamoorthi, R., \& Seetharaman, K. 2007. Image compression based on a family of stochastic models. Signal Processing, 87(3), 408-416.

Laba, M., Downs, R., Smith, S., Welsh, S., Neider, C., White, S., . . Baveye, P. 2008. Mapping invasive wetland plants in the hudson river national estuarine research reserve using quickbird satellite imagery. Remote Sensing of Environment, 112(1), 286-300.

Laba, M., Blair, B., Downs, R., Monger, B., Philpot, W., Smith, S., Baveye, P. C. 2010. Use of textural measurements to map invasive wetland plants in the hudson river national estuarine research reserve with IKONOS satellite imagery. Remote Sensing of Environment, 114(4), 876886.

Lee, K., Lunetta, R. 1996. Wetlands detection methods. In: Lyon, J., McCarthy, J. (Eds.), Wetland and environmental application of GIS. Lewis Publishers, New York. pp 249-275. 
Malthus, T. J., George, D. G. 1997. Airborne remote sensing of macrophytes in cefni reservoir, anglesey, UK. Aquatic Botany, 58(3-4), 317-332.

Matthews, E., Fung, I. 1987. Methane emission from natural wetlands: Global distribution, area,

McFeeters, S.K. 1996. The use of Normalized Difference Water Index (NDWI) in the delineation of open water features. International Journal of Remote Sensing, 17(7), 1425-1432.

National Wetlands Working Group. 1987. The canadian wetland classification system, provisional edition. ecological land classification series, no. 21. Ottawa, Ontario: Canadian Wildlife Service, Environment Canada.

Pasqualini, V., Pergent-Martini, C., Pergent, G., Agreil, M., Skoufas, G., Sourbes, L., \& Tsirika, A. 2005. Use of SPOT 5 for mapping seagrasses: An application to posidonia oceanica. Remote Sensing of Environment, 94(1), 39-45.

PCI Geomatics. 2010. PCI geomatica software: Release 10. Richmond Hill, Ontario, Canada.

Proctor, C., Robinson, V., He, Y. 2010. Multispectral detection of European Frog-bit in the south nation river using quickbird imagery. M.Sc. Thesis, University of Toronto.

Proctor, C., Robinson, V., He, Y. 2012. Multispectral detection of European Frog-bit in the South Nation River using Quickbird imagery. Canadian Journal of Remote Sensing, 38(4), 1-11.

Qi, J., Chehbouni, Al., Huete, A. R., Kerr, Y. H., Sorooshian, S. 1994. A modified soil adjusted vegetation index (MSAVI). Remote Sensing of Environment, 48(2), 119- 126.

R Development Core Team. 2008. R: A language and environment for statistical computing.

641 Fingerprints of global warming on wild animals and plants. Nature, 421(6918), 57-60. 
Sawaya, K. E., Olmanson, L. G., Heinert, N. J., Brezonik, P. L., \& Bauer, M. E. 2003. Extending satellite remote sensing to local scales: Land and water resource monitoring using highresolution imagery. Remote Sensing of Environment, 88(1-2), 144-156. 567). Thousand Oaks, CA: SAGE Publications, Inc.

Treitz, P., Howarth, P. 2000. High spatial resolution remote sensing data for forest ecosystem classification: An examination of spatial scale. Remote Sensing of Environment, 72(3), 268-289.

Vincent, G., Sabatiera, D., Blancb, L., et al. 2012. Accuracy of small footprint airborne LIDAR in its predictions of tropical moist forest stand structure. Remote Sensing of Environment, 125, 23-33.

Xu, M., Watanachaturaporn, P., Varshney, P. K., \& Arora, M. K. 2005. Decision tree regression for soft classification of remote sensing data. Remote Sensing of Environment, 97(3), 322-336

Yarbrough, L.D., Easson, G., Kuszmaul, J.S. 2005 Qucikbird 2 tasseled cap transformation coeffecients: A comparison of derivation methods. Pecora 16. Global Priorities in Land Remote Sensing, Sioux Falls, South Dakota, October 23-27.

Zhang, X. 1998. On the estimation of biomass of submerged vegetation using Landsat thematic mapper (TM) imagery: A case study of the Honghu Lake, PR China. International Journal of Remote Sensing, Vol. 19, No. 1, pp. 11-20. 
Table 1. Classification schema

\begin{tabular}{llll}
\hline Class Name & Abbreviation & Class Type & Class (\# of species) \\
\hline European Frog-bit & EFB & Species Level & Floating macrophyte (1) \\
Fragrant Water-Lily & FWL & Species Level & Floating macrophyte (1) \\
Submerged & Sub & Land Cover & Submerged macrophytes (2) \\
Duckweed & DW & Species Level & Floating macrophyte (1) \\
Grasses, Sedges, Rushes & GSR & Land Cover & Terrestrial grasses (multiple) \\
Mixed Forest & MF & Land Cover & Terrestrial trees (multiple) \\
Open Water & OW & Land Cover & No vegetation \\
\hline
\end{tabular}

671

672 
Table 2. Samples per Training and Reference Data Sets

\begin{tabular}{lll}
\hline Class Name & Training & Reference
\end{tabular}

\begin{tabular}{lll}
\hline European Frog-bit & 92 & 47
\end{tabular}

Fragrant Water-Lily $\quad 64 \quad 28$

Submerged $\quad 50 \quad 26$

Duckweed $28 \quad 14$

Grasses, Sedges, Rushes $\quad 90 \quad 45$

Mixed Forest $\quad 90 \quad 45$

673

\begin{tabular}{lll} 
Open Water & 90 & 45 \\
\hline
\end{tabular}

674 
Table 3. List of textural and spectral vectors under investigation

\begin{tabular}{lllrl}
\hline & Data Source & Derivative & \# of Bands & Total Vectors \\
\hline Textural vectors & & & \\
& Multispectral bands & N/A & 4 & 52 \\
& Panchromatic band & N/A & 1 & 13 \\
& Multispectral bands & Tasseled Cap & 3 & 39 \\
& & Principal Components & 3 & 39 \\
& NDVI & 1 & 13 \\
& MSAVI & DVW & 1 & 13 \\
& & NDWIF & 1 & 13 \\
& & 1 & 13 \\
& & N/A & & 4 \\
& & N/A & 4 & 1 \\
& Multispectral bands & 1 & 4 \\
& Pansharpenatic band bands & N/A & & 3 \\
& Multispectral bands & Tasseled Cap & 4 & 3 \\
& & Principal Components & 3 & 1 \\
& & NDVI & 3 & 1 \\
& & MSAVI & 1 & 1 \\
& & DVW & 1 & 1 \\
\hline
\end{tabular}


Table 4. List of Haralick texture attributes derived in this study (Haralick et al., 1973).

\begin{tabular}{|c|c|c|}
\hline $\begin{array}{l}\text { Texture } \\
\text { Attribute }\end{array}$ & Equation & Description \\
\hline Homogeneity & $t_{h o m}=\sum_{i, j=0}^{N_{g}-1} \frac{1}{1+(i-j)^{2}} p(i, j)$ & $\begin{array}{l}\text { The deviation from the } \\
\text { main diagonal. }\end{array}$ \\
\hline Contrast & $t_{c o n}=\sum_{i, j=0}^{N_{g}-1} p(i, j)(i-j)^{2}$ & $\begin{array}{l}\text { Local variation, increases } \\
\text { exponentially from } \\
\text { diagonal. }\end{array}$ \\
\hline Dissimilarity & $t_{d i s s}=\sum_{i, j=0}^{N_{g}-1} p(i, j)|i-j|$ & $\begin{array}{l}\text { Local variation, increases } \\
\text { linearly from diagonal. }\end{array}$ \\
\hline Mean & $t_{\text {mean }}=\sum_{i, j=0}^{N_{g}-1} i * p(i, j)$ & $\begin{array}{l}\text { Average grey level in the } \\
\text { local window }\end{array}$ \\
\hline Variance & $p(i, j) *\left(1-\mu_{i}\right)^{2}$ & $\begin{array}{l}\text { Measure of dispersion } \\
\text { around the central pixel. }\end{array}$ \\
\hline Entropy & $(i, j) \log (p(i, j))$ & $\begin{array}{l}\text { The randomness of the } \\
\text { intensity distribution. }\end{array}$ \\
\hline $\begin{array}{l}\text { Angular 2nd } \\
\text { Moment }\end{array}$ & $g^{-1} p(i, j)^{2}$ & $\begin{array}{l}\text { Local homogeneity that is } \\
\text { low when entries are almost } \\
\text { equal. }\end{array}$ \\
\hline Correlation & $t_{c o r r}=\sum_{i, j=0}^{N_{g}-1} \frac{p(i, j) *\left(1-\mu_{i}\right) *\left(1-\mu_{j}\right)}{\sqrt{\left(\sigma_{i}-\sigma_{j}\right)}}$ & $\begin{array}{l}\text { Measures the linear } \\
\text { dependency of grey levels. }\end{array}$ \\
\hline $\begin{array}{l}\text { GLDV Angular } \\
\text { 2nd Moment }\end{array}$ & $t_{G_{-} \text {ang }}=\sum_{i, j=0}^{N_{g}-1} p \delta(i)^{2}$ & $\begin{array}{l}\text { Measures local } \\
\text { homogeneity. }\end{array}$ \\
\hline GLDV Entropy & $p \delta(i) \log (p \delta(i))$ & $\begin{array}{l}\text { The opposite of GLDV } \\
\text { Angular Second Moment }\end{array}$ \\
\hline GLDV Mean & $t_{G_{-} \text {mean }}=\sum_{i, j=0}^{N_{g}-1} i * p \delta(i)$ & $\begin{array}{l}\text { Mathematically similar to } \\
\text { Dissimilarity }\end{array}$ \\
\hline GLDV Contrast & $t_{G_{-} \text {cont }}=\sum_{i, j=0}^{N_{g}-1} i^{2} * p \delta(i)$ & $\begin{array}{l}\text { Mathematically similar to } \\
\text { Contrast }\end{array}$ \\
\hline $\begin{array}{l}\text { Inverse } \\
\text { Distance }\end{array}$ & $t_{\text {invd }}=\sum_{i, j=0}^{N_{g}-1} \frac{p(i, j)}{|i-j|^{2}}$ for $i !=j$ & $\begin{array}{l}\text { Measures image } \\
\text { homogeneity. }\end{array}$ \\
\hline
\end{tabular}


678

Table 5. Semivariogram range at sill (\# of pixels)

\begin{tabular}{lcc}
\hline Class Name & $\begin{array}{c}\text { Multispectral Band } \\
\text { (band average) }\end{array}$ & Panchromatic Band \\
\hline European Frog-bit & 6.44 & 18.91 \\
Fragrant Water-Lily & 4.09 & 8.96 \\
Submerged & 6.66 & 15.25 \\
Duckweed & 6.54 & 10.80 \\
Grasses, Sedges, Rushes & 8.51 & 16.80 \\
Mixed Forest & 3.25 & 10.39 \\
Open Water & 6.29 & 25.30 \\
\hline
\end{tabular}

679

680 
Table 6. Jefferies-Matusita distance through forward selection

\begin{tabular}{lll}
\hline Iteration & Vector(s) & Distance \\
\hline 1 & Red & 1.47 \\
2 & Red + NIR & 1.87 \\
3 & Red + NIR + panchromatic dissimilarity & 1.97 \\
4 & Red + NIR + panchromatic dissimilarity & \\
& + tasseled cap band 1 contrast & 1.98 \\
\hline
\end{tabular}


682

Table 7. Accuracy statistics of the three decision tree classifications.

\begin{tabular}{lrrrrrr}
\hline & \multicolumn{2}{c}{ Iteration 2 } & \multicolumn{2}{c}{ Iteration 3 } & \multicolumn{2}{c}{ Iteration 4 } \\
\hline & UA & PA & UA & PA & UA & PA \\
EFB & 95.0 & 89.3 & 100.0 & 87.2 & 100.0 & 87.2 \\
FWL & 82.5 & 81.2 & 85.0 & 90.4 & 77.9 & 82.8 \\
DW & 86.2 & 89.3 & 96.5 & 100.0 & 100 & 100.0 \\
Species Only & & 87.3 & & 93.1 & & 91.3 \\
Overall Acc. & & 91.9 & 94.1 & \multicolumn{2}{c}{91.5} \\
Kappa & & 90.4 & 93.0 & \multicolumn{2}{c}{89.8} \\
\hline
\end{tabular}

683 


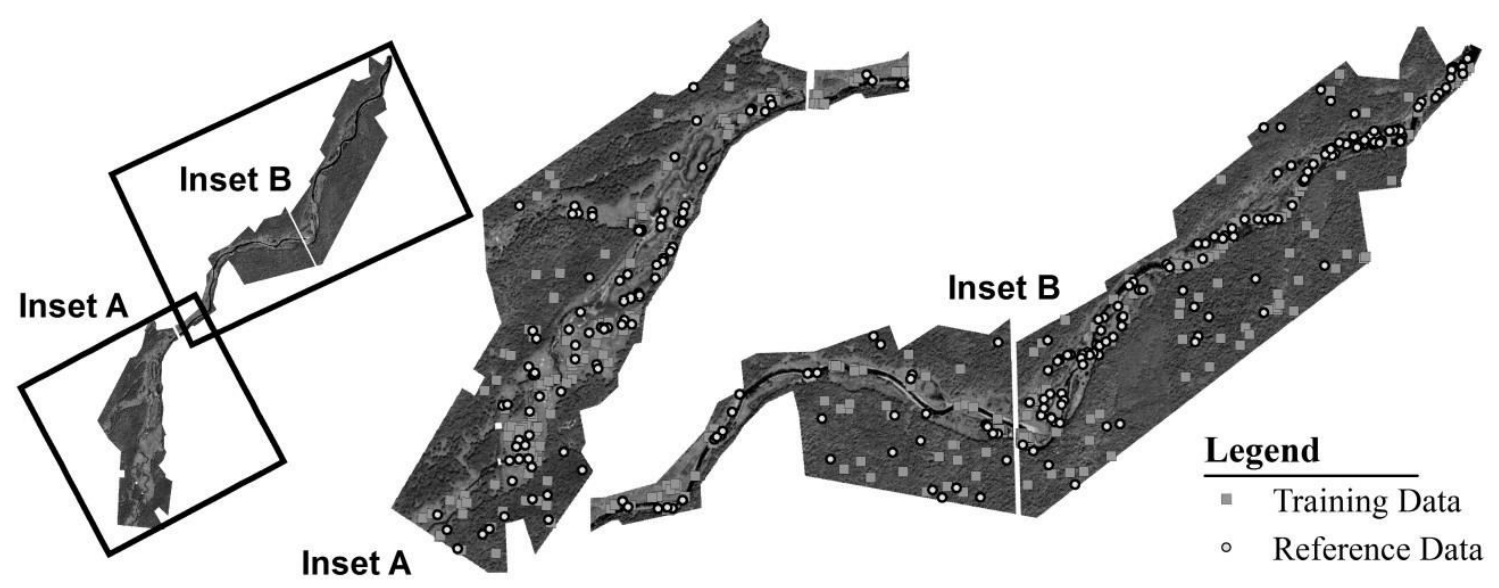

Figure 1. False color Quickird imagery of study area and the spatial location of the training and reference data sets, shown on two insets at 30:000 scale.

684

685

686

690

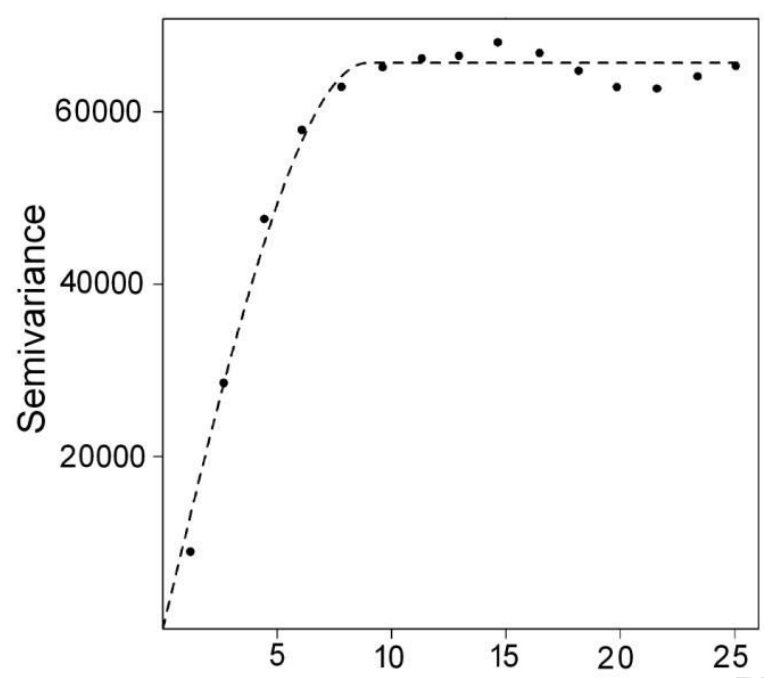

Pixel Distance

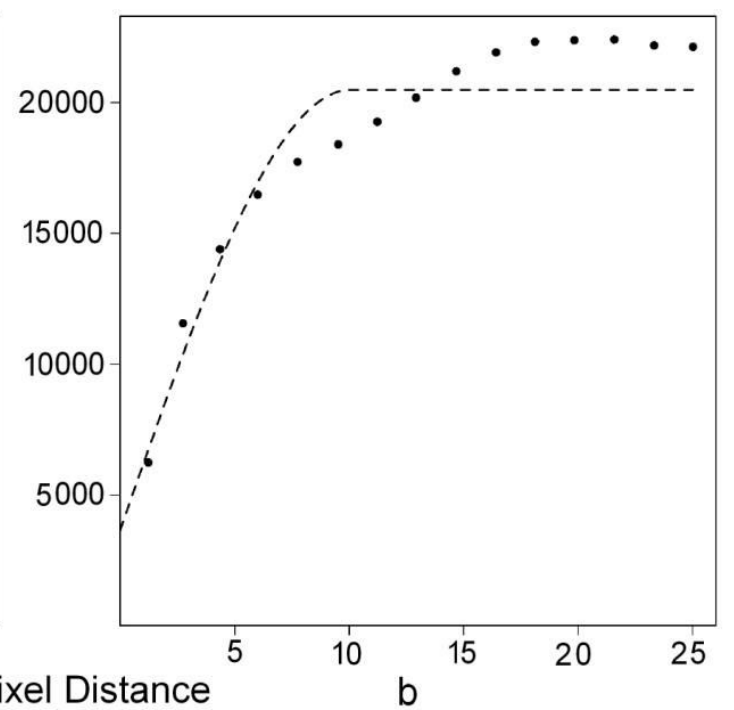

Figure 2. Semivariograms for panchromatic band with fitted models for the Fragrant Water-lily class (a) and the European Frog-bit class (b) 


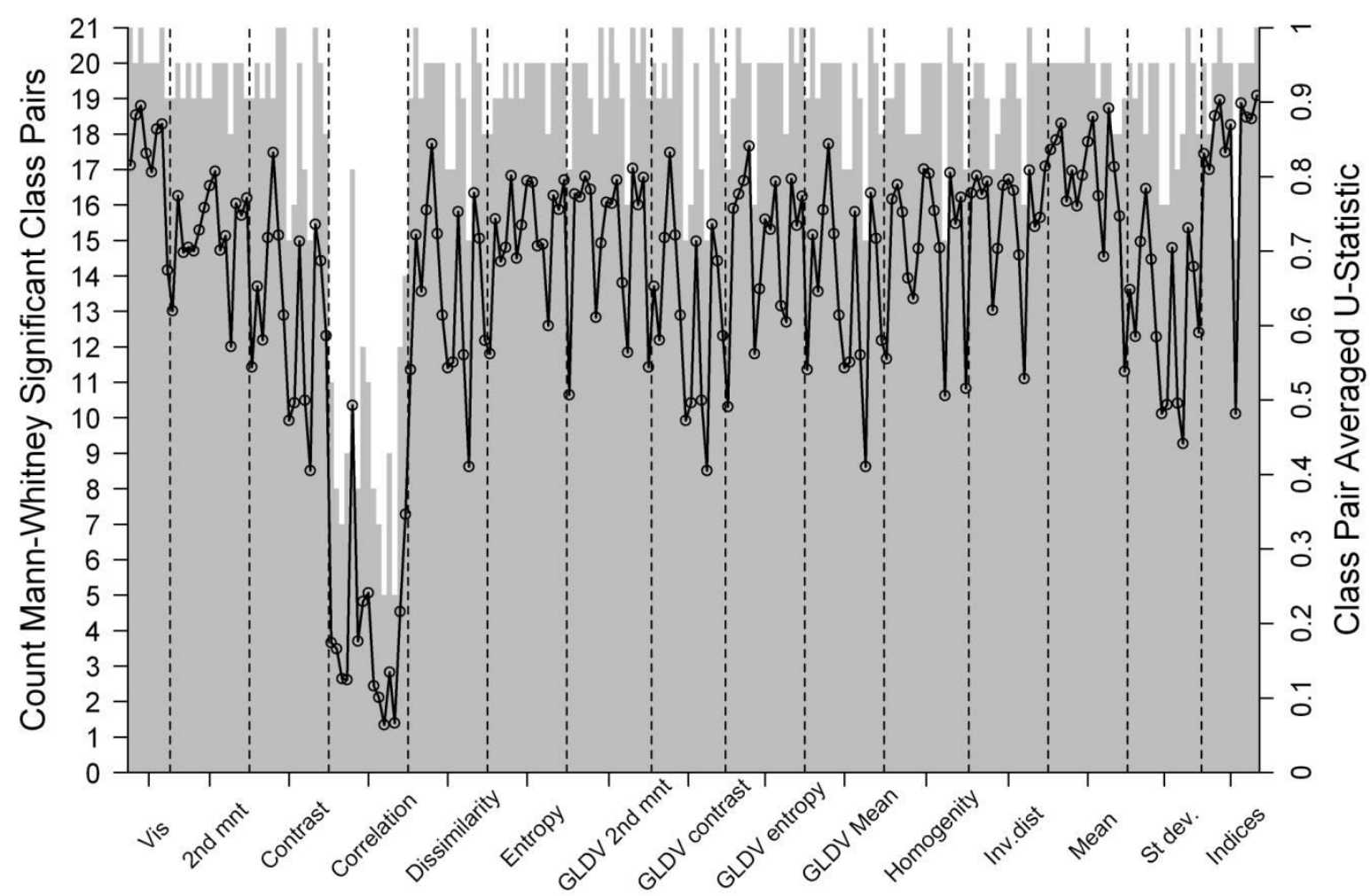

Figure 3. Plot of the one-dimensional distance measures. The grey columns are the results of the Mann-Whitney U-test expressed as the number of significant class pairs (left axis). The line indicates the average $\mathrm{U}$-Statistic distance measure for all class pairs (right axis). 
692

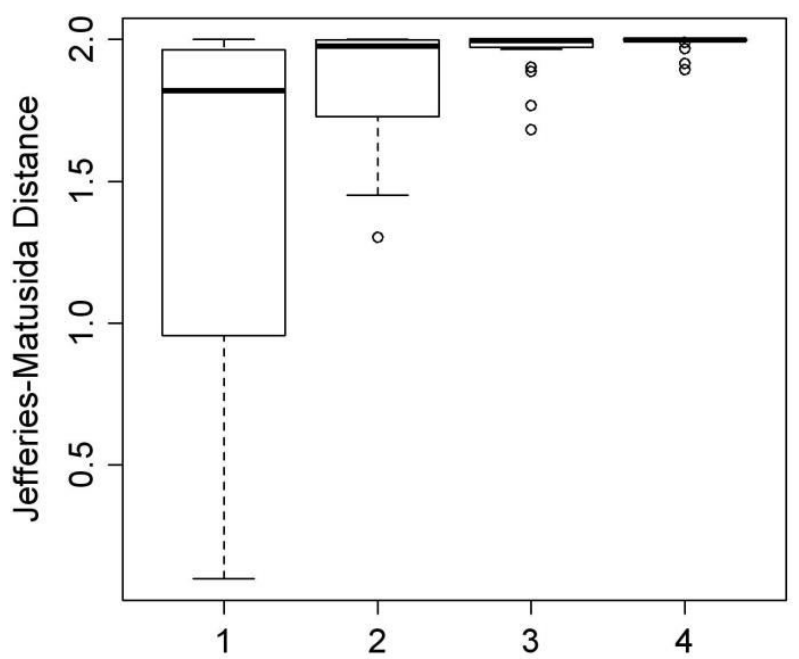

693

Interation

694

Figure 4. Jefferies-Matusita distance versus 695 forward selection iteration.

696 


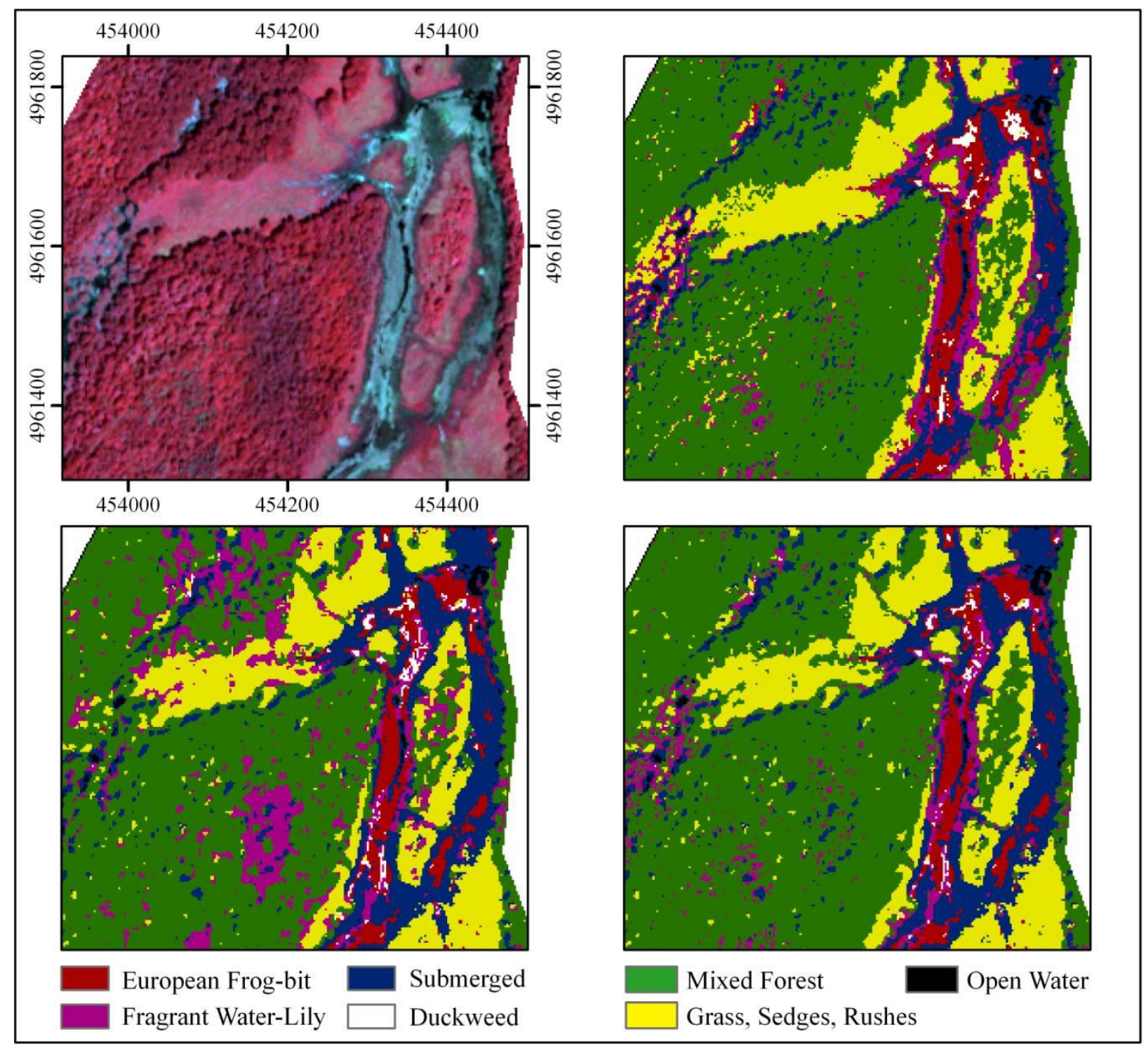

Figure 5. Decision trees classifications using Red/NIR bands (top right), Red/NIR/panchromatic dissimilarity (bottom right) and Red/NIR/panchromatic dissimilarity/Tasseled Cap brightness contrast (bottom left). False color Quickbird imagery (top left). 


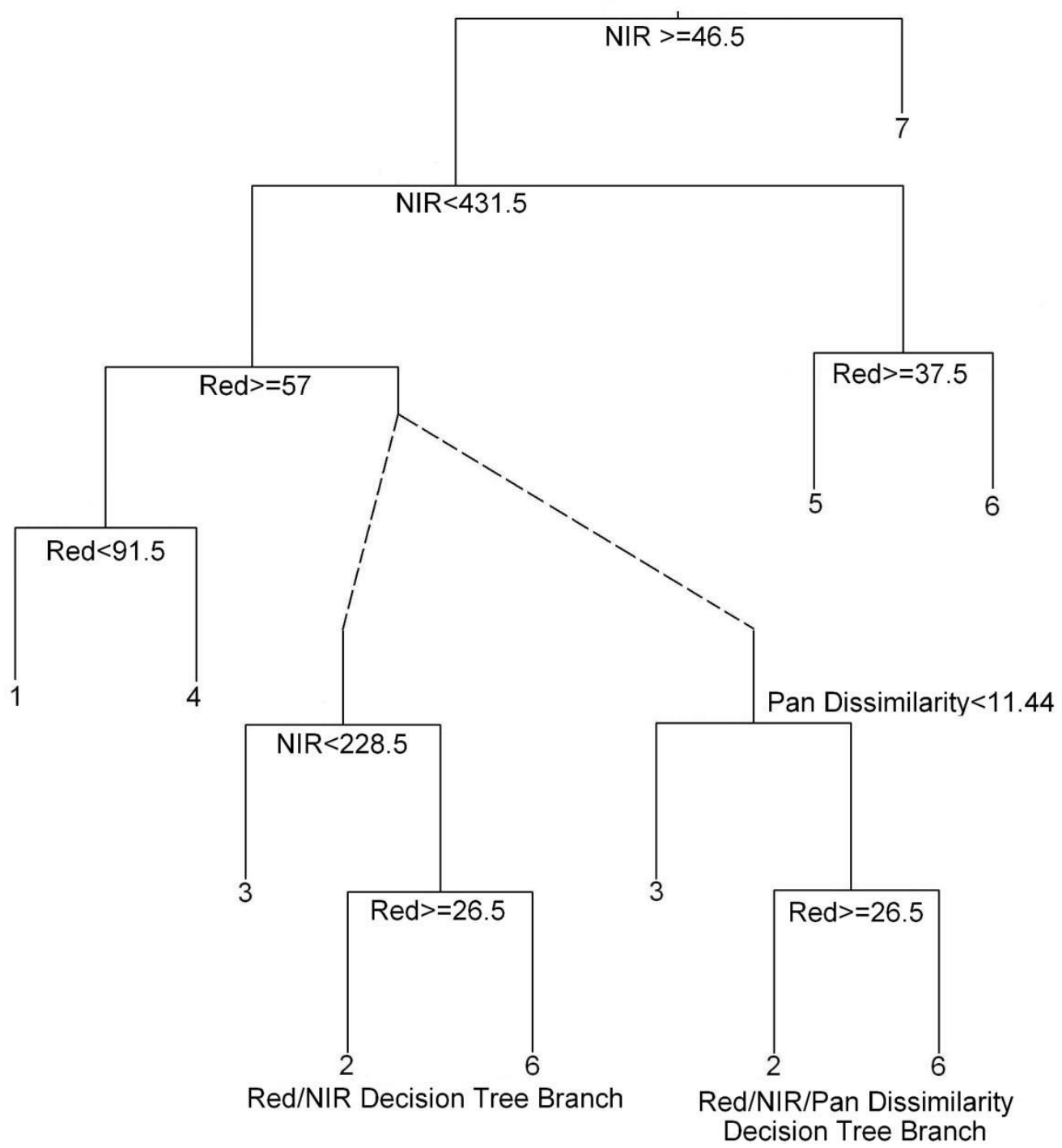

Figure 6. Decision tree indicating the similarity and divergence of the second and third iteration. 